\title{
Li-York Chaos of Set-valued Discrete Dynamical Systems Based on Semi-group Actions
}

\author{
QIAN Yun ${ }^{1}$ \\ Department of Mathematics \\ Chaohu University \\ Chaohu, China \\ e-mail: qianyun@chu.edu.cn
}

\author{
GUAN Peng ${ }^{2}$ \\ Department of Mathematics \\ Chaohu University \\ Chaohu,China \\ e-mail:guanpeng1983@163.com
}

\begin{abstract}
It is well known that a semi-group's action on a space could appear chaos phenomenon, like Li-York chaos and so on. Li-York chaos has important relations with topological transitivity and periodic point. This study analyzed metric space $(X, d)$ and it's dinduced Hausdorff metric space $(k(X), H)$. Let $T$ is a semi-group. We make $T$ continuously act on space $(X, d)$. We study topological transitivity and between $(X, d)$ and $(k(X), H)$. Some important results are presented which show that if is topological transitivity and periodicity (which means Li-York chaos at the same time), then the action of semigroup $T$ on $(k(X), H)$ is Li-York chaos.
\end{abstract}

Keywords-semi-group Actions; set-valued discrete dynamical systems; Li-York chaos; topological transitivity; Hausdorff metric

\section{INTRODUCTION}

Phenomenon of Chaos was found as early as 1903 in the beginning of this century, American mathematician J.H.Poincare in his book "Science and Method" proposed the Poincare Conjecture. Dynamical systems and topology of the two major areas are combined in his book, which pointed out that the possibility of a chaotic existence, thus becoming the world's first man who understands the possibility of chaos existence. In 1963, meteorologist E.Lorenz in Atmospheric Science magazine published an article of "decisive non-periodic flow", which pointed out that a link must exist between the powerless of weather forecasters and the climate could not accurately repeat. These studies clearly described the chaos state of sensitivity dependence on initial conditions. This is the famous butterfly effect.

For chaos research upsurge up in 1975, all kinds of definitions of chaos come into being $[1,2,3]$. Some classic definitions are Li-Yorke chaos [1], Devaney chaos [2] and chaotic group [3], and so on. Nowadays, study on chaotic semigroup focus on two aspects. One is the research on definition of chaotic semigroup action, include Li-Yorke chaotic semigroup action [6,7], Devaney chaotic semigroup

Anhui university provincial natural science research project (KJ2012A205) action [8], spatiotemporal chaotic semigroup action( ST chaos in short ) and study on the relationship between several chaotic definition. Such as semigroup action Devaney chaos is Stronger than Li - Yorke chaos[7], topologically strong mixing implies devaney chaos when semigroup continuously acts on the inverse limit space. The other is research on conditions of. In the article [5], they use Li-Yorke pairs to describe chaotic semigroup and discuss the condition of Li-Yorke pairs beingness. Article [8] study the affect of Sensitive Dependence on Initial of Chaotic semigroup actions. It is showed that Based on Li-Yorke pairs, the definition of spatiotemporal chaos of semigroup actions was given. Using Furstenberg families, w e studied the relations among the spatiotemporal chaos, mixing, and 2-rigidity, and we proved that topologically weakly mixing system implies ST chaos in the article [4].

This article would study some important natures of Setvalued Discrete Dynamical Systems.

\section{PRELIMINARIES}

Definition1[10]. Let $T$ be a semi-group, $X$ be a compact metric space. We say that $T$ continuously acts on $X$, if $\pi: T \times X \rightarrow X$ Satisfies the following two conditions:

(1) $\pi$ is continuous,

(2) $\pi(t, \pi(s, a))=\pi(t s, a), \forall t, s \in T, \forall a \in X$

Remark1. For convenience, we let $\pi(t, a)=t a$. So the condition (2) transform to $t(s a)=(t s) a, \forall t, s \in T$

,$\forall a \in X$. In this sense, the continuous action of semigroup $T$ on $X$ is equivalence with a semigroup homomorphism $\alpha: S \rightarrow C(X, X)$, where $C(X, X)$ is a semi-group composed by all continuous self-maps on $X$.

Definition2[10]. Suppose that a semi-group $T$ acts continuously on a compact metric space $X$. The subset $T(x)=\{t x \mid t \in T\}$ is called orbit with action of semigroup $T$ on each point $x \in X$.

Definition3[10]. Suppose that a semi-group $T$ acts continuously on a compact metric space $X$. If for every pair of none-empty open subsets $\mathrm{U}$ and $\mathrm{V}$ in $X$, there is 
some $t \in T$, such that $t(U) \cap V \neq \varnothing$.Then we say that the action of semi-group $S$ on $X$ is topologically transitive, which $t(U)=\{t u \mid u \in U\}$.

Definition4[11]. Suppose that $\pi: T \times X \rightarrow X$ is a continuous action. Define mapping on $k(X)$ that $\bar{\pi}: k(X) \times T \rightarrow k(X), \bar{\pi}(A)=\{\pi(a, T) \mid a \in A\}$. If there have $\bar{\pi}\left(t_{1}, \bar{\pi}\left(t_{2}, K\right)\right)=\bar{\pi}\left(t_{1} t_{2}, K\right)$, for each $K \in K(X)$ and every pair $t_{1}, t_{2} \in T$, then we call $(K(X), T, \bar{\pi})$ is a set-value dynamical system, $(K(X), T)$ in short.

Lemma1[5]. Suppose that $(X, T)$ is a topologically transitive dynamical system, $X$ is a Infinite compact metric space , $T$ is commutative semi-group. If $(X, T)$ has periodic point, then $(X, T)$ has Infinite scrambled set, and $(X, T)$ is called Li-Yorke chaos.

Lemma2[15]. Suppose that $X$ and $Y$ are two topological spaces and $f: X \rightarrow Y$ is a continuous mapping. If set $A$ is a compact set in space $X$, then $f(A)$ is a a compact set in space $Y$.

Lemma3[15]. $(k(X), H)$ is compact Metric space if and only if when $(X, d)$ is compact Metric space.

We definite the orbit of every point $x \in X$ is $\operatorname{orb}(x, T)=\{t x: t \in T\}$.

Suppose that $A$ is nonvoid constant closed subset of space $X$, if $A$ don't Include nonvoid proper constant closed subset, then we call $A$ minimal set. If $\operatorname{orb}(x, T)=\{t x: t \in T\}$ is a finite minimal set of $(X, T)$, we say $X$ is a periodic point of $(X, T)$.

\section{III.MAIN RESULTS}

Proposition1. Suppose that $A$ is a non-empty open subsets in space $X$. If $B \in k(X)$ and $B \subset A$, then there have:

(1)There have some $\varepsilon>0$, such that $N(B, \mathcal{\varepsilon}) \subset A$;

(2 $N(U)=\{B \mid B \in k(X), B \subset U\}$ constitutes all open subsets $\operatorname{in}\left(k(X), \tau_{H}\right)$ when $U$ is an open subset in space $X$.
Remark1. $\left(k(X), \tau_{H}\right)$ is topological space which is induced by Hausdorff Metric $H$ of $k(X)$.

Proof:

(1) It is clear that the conclusion is the fact in metric $\operatorname{space}(X, d)$.

(2)One side, we need to proof $N(U)$ is open subset of $\left(k(X), \tau_{H}\right)$ for every open subset $U$ in metric space $X$.

If we give every subset $B \in N(U)$, there have some $\varepsilon>0$, such that $N(B, \varepsilon) \subset U$. In $k(X)$ with the Hausdorff metric $\mathrm{H}$, we take neighbourhood $V(B, \varepsilon)$. We only proof that there is some $E \in N(U)$ for each $E \in V(B, \varepsilon)$. In fact, if $E \in V(B, \varepsilon)$, then it must be $H(B, E)<\varepsilon$. By the definition of $\mathrm{H}$, $E \subset N(B, \varepsilon) \subset U$, which means $E \in N(U)$. So $N(U)$ is open subset of $\left(k(X), \tau_{H}\right)$.

On the other side, for each open subset $K$ of $\left(k(X), \tau_{H}\right)$, we give every set $A \in K$. Because $A$ is compact set in space $X$, so there have finite open covering $\left\{U_{1}, \ldots U_{n}\right\}$, such that $A \subset U=\bigcup_{i=1}^{n} U_{i}$. Then we get some $\varepsilon_{0}>0$ that $N\left(A, \varepsilon_{0}\right) \subset U$ and $V\left(A, \varepsilon_{0}\right) \subset K$. Further, there have $H(E, A)<\varepsilon_{0}$ for each $E \in V\left(A, \varepsilon_{0}\right)$. By the definition of $\mathrm{H}$, we get $E \subset N\left(A, \varepsilon_{0}\right) \subset U$, which means $\quad A \in N(U)$

So $N(U)=\{B \mid B \in k(X), B \subset U\}$, thus we complete the proof. $\square$

Proposition2. If $\pi: T \times X \rightarrow X$ is a continuous action, then $\bar{\pi}: k(X) \times T \rightarrow k(X)$ is a continuous action too.

Proof: We first proof that $\bar{\pi}: k(X) \times T \rightarrow k(X)$ is self-mapping. It is clear that $\bar{\pi}(A)=\pi(A)$, for every $A \in k(X)$. By lemma2, we get $\pi(A)$ is compact subset of $A$, so that $\pi(A) \in k(X)$. For each set $B \in k(X)$, set $B$ is compact, so there some finite open covering $\left\{U_{1}, \ldots U_{n}\right\}$, such that $B \subset U=\bigcup_{i=1}^{n} U_{i}$. Because 
$\pi^{-1}(B) \subset \pi^{-1}(U)=\pi^{-1}\left(\bigcup_{i=1}^{n} U_{i}\right)=\bigcup_{i=1}^{n} \pi^{-1}\left(U_{i}\right)$ and $\quad \pi \quad$ is continues, so $\pi^{-1}\left(U_{i}\right)$ is open set, then $\pi^{-1}(B)$ has finite open covering in space $X$. So we get $\pi^{-1}(B)$ is compact of space $X \quad$ Last, there have $\bar{\pi}\left(\pi^{-1}(B)\right)=\pi\left(\pi^{-1}(B)\right)=B \in k(X) \quad, \quad$ so that $\bar{\pi}: k(X) \times T \rightarrow k(X)$ is self-mapping.

Next, we proof $\bar{\pi}$ is continue. It is easily to see $\bar{\pi}^{-1}(N(U))=\bar{\pi}^{-1}(\underset{\substack{B \subset U \\ B \in k(X)}}{U} B)=\underset{\substack{B \subset U \\ B \in k(X)}}{U} \pi^{-1}(B)=N\left(\pi^{-1}(U)\right)$ for each open set $N(U)$ in $k(X)$. Because $\pi$ is continue, so $\pi^{-1}(U)$ is open set of $X$. By the Proposition1, we get $N\left(\pi^{-1}(U)\right)$ is open set of $k(X)$, so $\bar{\pi}$ is continue.

Theorem1. $\bar{\pi}: k(X) \times T \rightarrow k(X)$ is topological transitive if and only if $\pi: T \times X \rightarrow X$ is topological transitive.

Proof: suppose that pair of none-empty open sets $U$ and $V$ in $X$, then $N(U)$ and $N(V)$ are none-empty open sets of $k(X)$. By definite of topological transitive, there have some $t \in T$, such that $t(N(U)) \cap N(V) \neq \varnothing$.

Because

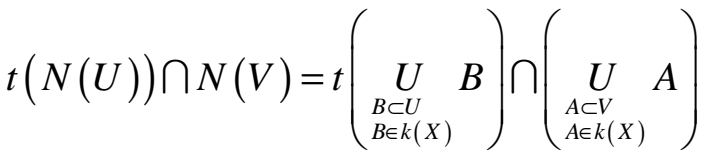

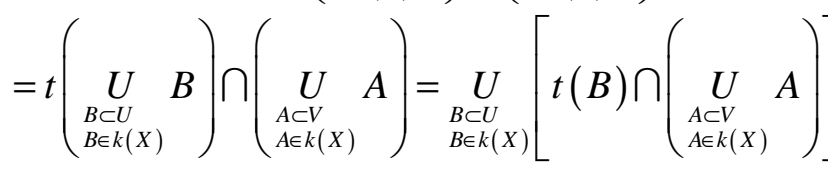

$$
\begin{aligned}
& =\underset{\substack{B \subset U \\
B \in k(X)}}{U} \underset{\substack{A \subset V \\
A \in k(X)}}{U}[t(B) \cap A] \neq \varnothing
\end{aligned}
$$

, so there must be some sets $A \subset V$ and $B \subset U$, such that $t(B) \cap A \neq \varnothing \Rightarrow t(U) \cap V \neq \varnothing$. Then we proof that $\pi: T \times X \rightarrow X$ is topological transitive.

Next, we suppose $\pi: T \times X \rightarrow X$ is topological transitive. Then for every pair of none-empty open subsets $U$ and $V$ in $X$, there is some $t \in T$, such that $t(U) \cap V \neq \varnothing$. We take arbitrary pair $M, N \subset K(X)$. Let

$$
\begin{gathered}
M=M\left(U_{1}, U_{2}, \ldots, U_{n}\right)=\left\{E \in K(X) \mid E \subset \bigcup_{i=1}^{n} U_{i}, E \cap U_{i} \neq \varnothing\right\} \\
N=N\left(U_{1}, U_{2}, \ldots, U_{n}\right)=\left\{F \in K(X) \mid F \subset \bigcup_{i=1}^{n} U_{i}, F \cap U_{i} \neq \varnothing\right\}
\end{gathered}
$$

Then $s(M) \cap N=\{s E \mid E \in M\} \cap\{F \mid F \in N\}$, so there have some $U_{k_{1}} \in\left(U_{1}, U_{2}, \ldots, U_{n}\right)$ and some $U_{k_{2}} \in\left(U_{1}, U_{2}, \ldots, U_{n}\right)$, such that $s\left(U_{k_{1}}\right) \cap U_{k_{2}} \subset\{s E \mid E \in M\} \cap\{F \mid F \in N\}$. Because $U_{k_{1}}$ and $U_{k_{2}}$ are none-empty open subsets, so $s\left(U_{k_{1}}\right) \cap U_{k_{2}} \neq \varnothing \quad$, then $s(M) \cap N \neq \varnothing \quad, \quad$ so $\bar{\pi}: k(X) \times T \rightarrow k(X)$.

The following theorem would discuss Li-York chaos about $\bar{\pi}$. Suppose that $\phi(X)$ is subspace of $k(X)$, which is formed by all single point sets of space $X$. It is easy to see $\left(X, \tau_{d}\right)$ and $\left(\phi(X), \tau_{H}\right)$ is homeomorphous.

Theorem2. Suppose that $E$ is non-void finite set, $E$ is a periodic point of system $(K(X), T)$ if and only if every point of $A$ is periodic point of system $(X, T)$.

Proof:

$\Rightarrow$ Suppose $E$ is a periodic point of system $(K(X), T)$, then there must be some $t \in T$, such that $t(E)=E$. So we can say the continuous action of $\bar{\pi}$ on space $K(X)$ means equivalent replacement, so every point of $A$ is periodic point of system $(X, T)$.

$\Leftarrow$ Suppose $E=\left\{x_{1}, x_{2}, \ldots, x_{n}\right\}$ and $x_{i}$ is periodic point of $\operatorname{system}(X, T)$. So $\operatorname{orb}\left(x_{i}, T\right)$ is finite minimal ]$_{\text {set, }}$ then $\left\{t x_{1}, t x_{2}, \ldots, t x_{n}\right\}$ is finite minimal set for each $t \in T$. By lemma3, $(k(X), H)$ is compact metric space. So $A$ is a periodic point of system $(K(X), T)$.

\section{IV.PROOF OF THEOREM 3}

Theorem3.If $\pi: T \times X \rightarrow X$ is topological transitivity and have periodic point, then $\bar{\pi}: k(X) \times T \rightarrow k(X)$ is Li-Yorke chaos.

Proof: because $\pi: T \times X \rightarrow X$ is topological transitivity and have periodic point, so by Theorem1 and Theorem $2, \bar{\pi}: k(X) \times T \rightarrow k(X)$ is topological transitivity and 
have periodic point too. So by Lemma1, $\bar{\pi}: k(X) \times T \rightarrow k(X)$ is Li-Yorke chaos.

\section{CONCLUSION}

In this paper, we We laid special stress on researching Li-York Chaos of Set-valued Discrete Dynamical Systems Based on Semi-group Actions. It woule be showen that when semigroup act on a compact metric space $(X, d)$, if the action is topological transitivity and have periodic point, then we will get the same result when this semigroup act on the space $(k(X), H)$ which is dinduced by metric $d$.

\section{REFERENCES}

[1] Li T.Y. and Yorke J . A . Period three implies chaos. Amer. Math. Monthly, 1975, (82): 985-992.

[2] Devanney R. An Introduction to Chaotic Dynamical Systems , Reading MA : Addison Wesley, 1989.

[3] Cairns G, Davis G, Elton D, et al . Chaotic Group Actions. Enseign. Math. 1995, 41: 123-133.

[4] Yan Xinhua, Ren Yunli. Some Remarks on the Spatiotemporal Chaos Behavior of Semigroup Actions. Journal of Beijing Inst itute of Petrochemical Technology.2011,19(1):57-59.

[5] SU Xun-li. On Li-Yorke pairs of semigroup actions. Pure and Applied Mathematics. 2010, 26 (4) : 608-613

[6] Su Xunli, Zhou youcheng. Chaotic semigroup actions. App1. Math. J. Chinese Univ. Ser. A 2004, 19(3): 292-296.

[7] SU Xun-li. Chaotic group actions . Journal of Zhejiang University(Science Edition)·2005, 32(1): $13 \sim 16$

[8] Peng Guan,Yun Qian.Sensitive Dependence on Initial of Chaoticsemigroup actions. 2012 8th International Conference on Natural Computation,2012,3: 957-959

[9] Qin Xiaolong, Zhou Youcheng. Mixing property and chaos in setvalued dynamical systems. University Applied mathematics Journa, 2008,23(1):121-126

[10] Liao Gongfu, Wang Lidong. A kind of set-valued mapping transitivity, mixed and chaos.SCIENCE IN CHINA Ser.A Mathematics.2005,35(10):1155-1161

[11]WANG Hu,i FAN Q in-jie. Topological Ergodicity, Entropy and Chaos of Set-valued Discrete Systems. JOURNAL OF JILIN UNIVERSITY ( SCIENCE EDITION ),2007,45(6):903-906

[12]Naolekar A C, Sankaran P. Chaotic group actions onmanifolds .Topology Appl., 2000, 107: 233 243.

[13] W.Huang, X.Ye, Homeomorphisms with the whole compacta being scrambled sets, in:Ergosic Theory Dynamical Systems, to appear.

[14]W.Huang, X.Ye,Devaney's chaos or 3-scattering implies Li-Yorke's chaos.Topology Appli., 2002, 117(3):259-272

[15] Heriberto R F. A Note on Transitivity in Set-valued Discrete Systems [J].Chaos , Solitions and Fractals, 2003, 17: 99-104

[16] Blanchard F, Host B, Maass A. Topological complexity. Ergod. Th. And Dynam. Sys., 2000, 20(3):641-662

[17] Akin E, Glasner E. Residual properties and almost equicontinuit.J.d'Anal.Math.,2001, 84: 243-286

[18] Glasner E, Weiss B. Locally equicontinuous dynamical systems. Colloq. Math.,2000,84-85 (2):345-361

[19] Yang R. Topological sequence complexity and mixing. Chinese . Ann. Math. Ser.A, 2004, 25 (6) :809-816

[20]Huang W, Ye XD. Topological complexity, return times and weak disjointness. Ergod. Th. And Dynam. Sys.,2004,24(3):825-846

[21]Dou D, Ye XD, Zhang GH. Entropy sequences and maximal entropy sets. Nonlinearity,2006, 19(1):53-74
[22] Srinivasan Gopal . Ying-Cheng Lai. Inducing Chaos in MOSFETBased Electronic Circuits Circuits Syst Signal Process (2009) 28: 535-545

[23] HuoyunWang, Xiongwu Long, Heman Fu. Sensitivity and chaos of semigroup actions. Semigroup Forum (2012) 84:81-90 